\title{
Scalable Alternating Projection and Proximal Splitting for Array Pattern Synthesis
}

\author{
Yubing Han and Chuan Wan \\ School of Electronic and Optical Engineering, Nanjing University of Science and Technology, Nanjing, Jiangsu 210094, China \\ Correspondence should be addressed to Yubing Han; hanyb@mail.njust.edu.cn
}

Received 2 July 2015; Revised 15 August 2015; Accepted 17 August 2015

Academic Editor: Felipe Cátedra

Copyright ( 2015 Y. Han and C. Wan. This is an open access article distributed under the Creative Commons Attribution License, which permits unrestricted use, distribution, and reproduction in any medium, provided the original work is properly cited.

\begin{abstract}
Array synthesis with embedded element patterns is a problem of great practical importance. In this paper, an array pattern synthesis method using scalable alternating projection and proximal splitting is proposed which considers the scaling invariance property of design specifications and constraints for the amplitudes of pattern and excitation. Under the framework of alternating projection, the scalable pattern and excitation constraint sets are first defined. Then the scalable pattern projection and iterative procedure for optimum pattern scaling factor are studied in detail. For the scalable excitation projection, it is designed as the solution to a constrained weighted least mean squares optimization, which can be solved by an effective forward-backward splitting iterative process. Finally, the selection of the weighted matrix and computational complexity are discussed briefly. Several typical linear and planar synthesis examples with or without the embedded element patterns are provided to demonstrate the effectiveness and power of the proposed method.
\end{abstract}

\section{Introduction}

The synthesis problem for array antennas can be loosely defined as the inverse of the analysis problem, that is, to determine the element excitations and possibly the lattice geometry, so that the array radiation pattern or the excitations satisfy some prescribed requirements. It is a vast subject in the antenna research, which has applications in fields, such as radar, sonar, radio astronomy, and satellite communications [1].

There are many methods to synthesize a desired pattern. The early history of the synthesis algorithm could date back to Woodward-Lawson method [2], which was firstly presented for linear arrays, and then extended to the circular and planar arrays. The synthesis methods in this stage established the basic pattern decomposition theory, using expanding characters of Fourier series and Bessel function and solving the question with some energy optimization ways. Yet, poor controls for side lobe levels (SLLs) and ripples in the desired coverage are the common problems for these early methods, so some algorithms using Taylor or Chebyshev distribution as aperture current distribution made a great effect on solving them next stage [3, 4]. However, these Taylor or Chebyshev synthesis methods have some drawbacks. The applied array should be a circle or rectangle, and this would be difficult to apply during most synthesis procedures. Today, iterative methods based on optimization techniques are very powerful tools for pattern synthesis thanks to the modern computer. There are many types of random optimization methods that have been applied to antenna synthesis problems, such as genetic algorithm, differential evolution, simulated annealing, and particle swarm optimization [5-8]. These optimization methods are computationally expensive and they are not effective in large arrays because a large number of particles must be needed in these situations.

Alternating projection (AP) is an extremely powerful and versatile procedure for synthesizing the excitation of very general antenna structures $[9,10]$. The synthesis problem is formulated as the search of the intersection of sets and is solved by an iterative projection method. On the other hand, the least mean squares (LMS) or weighted least mean squares (WLMS) method is popular in array synthesis [11], which formulates pattern of array in matrix form and minimizes the difference of realized pattern and desired pattern in LMS sense. A variation of the LMS method is described in [12], where different weights for different directions are introduced 
and the weights are changed iteratively according to the relative errors in each direction. In [13], a novel alternating adaptive projections (AAP) algorithm for antenna synthesis is presented based on the scaling invariance property of design specifications and constraints which arises in synthesis problems of practical relevance, and an iterative procedure is proposed to compute the optimum scale parameter (or reference level) which is simple to implement and easily integrated in standard alternating projection routines. In [14], a Gram-Schmidt orthogonalization method is proposed for the radiation pattern synthesis. It applies the GramSchmidt procedure to obtain an orthonormal basis from the embedded element patterns, and then the pattern synthesis is viewed as finding the projection on a linear space spanned by this basis, which can be solved by using WLMS optimization. Recently, an efficient synthesis method called the weighted alternating reverse projection (WARP) was developed in [15, 16], which combined the power of WLMS and alternating projection together by iteratively modifying the target pattern and weighting values of different directions.

With the inspiration of WARP, we have proposed an array synthesis method with consideration of embedded element patterns by using weighted alternating projection and proximal splitting [17], whose salient characteristic is that the projector over excitation constraint set is designed as the solution to a constrained weighted least mean squares (CWLMS) problem which can be efficiently solved by using proximal splitting method. In this paper, our alternating projection and proximal splitting method is extended to consider the scaling invariance property of design specifications and constraints (particularly for the amplitudes of pattern and excitation).

The rest of the paper is organized as follows. In Section 2, we present the problem statement for array synthesis with embedded element patterns. The proposed array pattern synthesis method is developed in Section 3. Several synthesis examples are reported in Section 4 to demonstrate the efficiency of the proposed method. Conclusions are drawn in Section 5 .

\section{Problem Statement}

Consider an arbitrary antenna array with $N$ elements. Suppose that the embedded pattern of the $n$th element with $K$ sampling directions $\left.\left(\theta_{k}, \varphi_{k}\right)\right|_{k=1} ^{K}$ is expressed as $\mathbf{x}_{n}=\left[x_{n}\left(\theta_{1}\right.\right.$, $\left.\left.\varphi_{1}\right), \ldots, x_{n}\left(\theta_{K}, \varphi_{K}\right)\right]^{T}$, where $\theta$ and $\varphi$ are the elevation and azimuth angles. The far field pattern obtained by the superposition of those embedded element patterns can be written as

$$
\mathbf{F}=\sum_{n=1}^{N} a_{n} \mathbf{x}_{n}=\mathbf{X} \mathbf{a},
$$

where $\mathbf{a}=\left[a_{1}, \ldots, a_{N}\right]^{T}$ is the array excitations, $\mathbf{F}=\left[f\left(\theta_{1}\right.\right.$, $\left.\left.\varphi_{1}\right), \ldots, f\left(\theta_{K}, \varphi_{K}\right)\right]^{T}$, and $\mathbf{X}=\left[\mathbf{x}_{1}, \ldots, \mathbf{x}_{N}\right]$. The purpose of array synthesis is to find a set of complex excitations so that the field pattern and excitations can meet the specified requirements.

Same as AAP method [13], we consider the scaling invariance property of design specifications and constraints for the amplitudes of pattern and excitation. The scalable pattern constraint sets are defined as

$$
\begin{gathered}
S_{\text {patt }}=\bigcup_{\alpha \neq 0} S_{\text {patt }}^{\alpha}, \\
S_{\text {patt }}^{\alpha}: \alpha M_{L}\left(\theta_{k}, \varphi_{k}\right) \leq\left|f\left(\theta_{k}, \varphi_{k}\right)\right| \leq \alpha M_{U}\left(\theta_{k}, \varphi_{k}\right), \\
1 \leq k \leq K,
\end{gathered}
$$

where $\alpha$ is arbitrary nonzero scaling parameter for pattern constraints and $\alpha M_{L}$ and $\alpha M_{U}$ are the upper and low masks. The scalable excitation constraint sets are represented as

$$
\begin{gathered}
S_{\mathrm{exc}}=\bigcup_{\beta \neq 0} S_{\mathrm{exc}}^{\beta}, \\
S_{\mathrm{exc}}^{\beta}:\left\{\begin{array}{l}
\beta A_{\min } \leq\left|a_{n}\right| \leq \beta A_{\max } \\
\phi_{\min } \leq \arg \left(a_{n}\right) \leq \phi_{\max },
\end{array} \quad 1 \leq n \leq N,\right.
\end{gathered}
$$

where $\left|a_{n}\right|$ and $\arg \left(a_{n}\right)$ are the amplitude and phase of $a_{n}, \beta$ is arbitrary nonzero scaling parameter, and $\beta A_{\min }, \beta A_{\max }, \phi_{\min }$, and $\phi_{\max }$ limit the amplitude and phase variations for each excitation. Obviously, for the case of phase-only array synthesis, we have $A_{\text {min }}=A_{\text {max }}$. It is worth noting that $S_{\text {patt }}$ and $S_{\text {exc }}$ may not be convex because they are the unions of some convex sets. The purpose of the array synthesis is to find the intersection of these pattern and excitation constraint sets.

\section{Proposed Array Pattern Synthesis Method}

3.1. The Method of Alternating Projections. According to the above discussion, the array synthesis problem can be formulated as the search of the intersection of constraint sets. Our solution is the method of AP $[18,19]$. Here we first briefly introduce the method of AP. Given $M$ sets $S_{1}, \ldots, S_{M}$ with $\bigcap_{i=1}^{M} S_{i} \neq \varnothing$, we seek a feasible solution $\mathbf{x}^{*} \in \bigcap_{i=1}^{M} S_{i}$ which belongs to the intersection of constraint sets. For the AP algorithm, the most important concept is the projection operator. The projector $\mathbf{P}_{A}$ over a subset $A$ is defined as the operator whose value of $\mathbf{P}_{A} \mathbf{x}$ is a point $\mathbf{y} \in A$ such that every point in $A$ has a distance from $\mathbf{x}$ not smaller than $\mathbf{y}$. Note that when $A$ is a nonconvex set, more than such a point can exist; in this case we assume that a rule of choice has been fixed. The $\mathrm{AP}$ algorithm can be expressed by the iterative process

$$
\mathbf{x}_{j+1}=\mathbf{P}_{M} \cdots \mathbf{P}_{1} \mathbf{x}_{j}
$$

where $j$ is the iteration time and $\mathbf{P}_{i}, i=1, \ldots, M$, are the $M$ projectors over the constraint sets $S_{i}, i=1, \ldots, M$. That is to say, the method of AP can be implemented by projecting onto the constraint sets cyclically in each iteration, with the desired point obtained in the limit. In this paper, a weighted $\mathrm{AP}$ algorithm is used in the application of array synthesis with scalable pattern and excitation constraints.

3.2. Scalable Pattern Projection. In this subsection, the scalable pattern projection over pattern constraint sets is discussed. We first define the projector over $S_{\text {patt }}^{\alpha}$ with a fixed scaling factor $\alpha$ by 


$$
\mathbf{P}_{\text {patt }}^{\alpha}: f\left(\theta_{k}, \varphi_{k}\right) \longrightarrow f^{\alpha}\left(\theta_{k}, \varphi_{k}\right)= \begin{cases}\alpha M_{U}\left(\theta_{k}, \varphi_{k}\right) \frac{f\left(\theta_{k}, \varphi_{k}\right)}{\left|f\left(\theta_{k}, \varphi_{k}\right)\right|}, & \left|f\left(\theta_{k}, \varphi_{k}\right)\right|>\alpha M_{U}\left(\theta_{k}, \varphi_{k}\right) \\ f\left(\theta_{k}, \varphi_{k}\right), & \alpha M_{L}\left(\theta_{k}, \varphi_{k}\right) \leq\left|f\left(\theta_{k}, \varphi_{k}\right)\right| \leq \alpha M_{U}\left(\theta_{k}, \varphi_{k}\right) \\ \alpha M_{L}\left(\theta_{k}, \varphi_{k}\right) \frac{f\left(\theta_{k}, \varphi_{k}\right)}{\left|f\left(\theta_{k}, \varphi_{k}\right)\right|}, & \left|f\left(\theta_{k}, \varphi_{k}\right)\right|<\alpha M_{L}\left(\theta_{k}, \varphi_{k}\right),\end{cases}
$$

where $\mathbf{F}^{\alpha}=\left[f^{\alpha}\left(\theta_{1}, \varphi_{1}\right), \ldots, f^{\alpha}\left(\theta_{K}, \varphi_{K}\right)\right]^{T}$. Then the projector over the scalable pattern constraints $S_{\text {patt }}$ can be defined as

$$
\mathbf{P}_{\text {patt }}: \mathbf{F} \longrightarrow \mathbf{F}^{\tilde{\alpha}}=\mathbf{P}_{\text {patt }}^{\tilde{\alpha}} \mathbf{F},
$$

where $\widetilde{\alpha}=\arg \min _{\alpha}\left\|\mathbf{F}-\mathbf{F}^{\alpha}\right\|_{x}$ and $\|\cdot\|_{x}$ is an arbitrary norm definition, such as $l_{2}, l_{1}$, and $l_{\infty}$ norms. In this paper, we select $l_{2}$ norm since the $l_{2}$ norm dynamic projector presents the most robust operation in terms of solution quality and convergence rate [13]. In each iteration, the optimum scaling factors $\widetilde{\alpha}$ can be determined by following Algorithm 1 . For convenience, we denote $\mathbf{F}_{r}=\left([\operatorname{Re}\{\mathbf{F}\}]^{T},[\operatorname{Im}\{\mathbf{F}\}]^{T}\right)^{T}$ and $\mathbf{F}_{r}^{\alpha}=$ $\left(\left[\operatorname{Re}\left\{\mathbf{F}^{\alpha}\right\}\right]^{T},\left[\operatorname{Im}\left\{\mathbf{F}^{\alpha}\right\}\right]^{T}\right)^{T}$, where $\operatorname{Re}\{\cdot\}$ and $\operatorname{Im}\{\cdot\}$ represent the real and imaginary parts of a complex vector, respectively.

Algorithm 1 (iterative procedure for optimum pattern scaling factor $\widetilde{\alpha})$.

Step 1. Initialize the scaling factor $\alpha$.

Step 2. Obtain $\mathbf{F}^{\alpha}$ by carrying on the projector $\mathbf{P}_{\text {patt }}^{\alpha}$.

Step 3. Calculate the relative error $\varepsilon=\left(\mathbf{F}_{r}^{\alpha}-\mathbf{F}_{r}\right)^{T} \mathbf{F}_{r}^{\alpha} /\left\|\mathbf{F}_{r}^{\alpha}\right\|_{2}^{2}$.

Step 4. Update the scaling factor by $\alpha=\alpha(1-\varepsilon)$.

Step 5. If convergent or equal to the maximum iteration time, then $\widetilde{\alpha}=\alpha$ and break; else return to Step 2 .

3.3. Scalable Excitation Projection. Now, we address the scalable excitation projection over excitation constraint sets. After we obtain a new pattern $\mathbf{F}$ by using projector $\mathbf{P}_{\text {patt, }}$, an appropriate way must be found to transform $\mathbf{F}$ to a new excitation $\mathbf{a}$. It can be implemented by minimizing the difference between the temporary field pattern $\mathbf{F}$ and synthesized radiation pattern $\mathbf{X a}$ under the excitation constraint $S_{\text {exc }}$, which can be solved by a constrained weighted LMS such that

$$
\mathbf{P}_{\text {CWLMS }}: \mathbf{F} \longrightarrow \underset{\mathbf{a} \in S_{\text {exc }}}{\arg \min } \frac{1}{2}\|\mathbf{F}-\mathbf{X a}\|_{\mathbf{W}}^{2}
$$

where $\mathbf{W}=\operatorname{diag}\left[w\left(\theta_{1}, \varphi_{1}\right), \ldots, w\left(\theta_{K}, \varphi_{K}\right)\right]$ is a diagonal matrix with weights for each direction; it can be adjusted according to the relative importance of the different directions of the temporary pattern. The details of choosing $\mathbf{W}$ will be given in Section 3.4.
To solve (7), a proximal splitting method is developed. We first introduce the proximity operator [20,21]; for a point $\mathbf{x}$ in set $A$, the minimization problem

$$
\min _{\mathbf{y} \in A}\left\{f(\mathbf{y})+\frac{1}{2}\|\mathbf{x}-\mathbf{y}\|^{2}\right\}
$$

admits a unique solution, which is denoted by $\operatorname{prox}_{f} \mathbf{x}$. The operator $\operatorname{prox}_{f} \mathbf{x}: A \rightarrow A$ thus defined is the proximity operator of $f$. Similar to the definition of projector $\mathbf{P}_{A}$, if there are multiple minima, we assume that a rule of choice has been fixed.

Obviously, (7) is equivalent to the following unconstrained problem:

$$
\mathbf{P}_{\text {CWLMS }}: \mathbf{F} \longrightarrow \underset{\mathbf{a}}{\arg \min }\left\{\ell(\mathbf{a})+\frac{1}{2}\|\mathbf{F}-\mathbf{X a}\|_{\mathbf{W}}^{2}\right\},
$$

where $\ell$ is the indicator function of $S_{\text {exc }}$ :

$$
\ell: \mathbf{a} \longrightarrow \begin{cases}0, & \mathbf{a} \in S_{\mathrm{exc}} \\ +\infty, & \mathbf{a} \notin S_{\mathrm{exc}} .\end{cases}
$$

It may not be convex because of the nonconvexity of $S_{\text {exc }}$. From [17, 20, 21], we can know that (9) can be effectively solved by using forward-backward splitting iterative process

$$
\mathbf{a}^{(q+1)}=\operatorname{prox}_{\ell} \mathbf{P}_{U} \mathbf{a}^{(q)},
$$

where $\mathbf{P}_{U} \mathbf{a}=\mathbf{a}+\gamma \mathbf{X}^{H} \mathbf{W}(\mathbf{F}-\mathbf{X a})$ is the forward gradient step, $q$ is the iteration time for solving (9), and $\gamma$ is a step-size parameter which can be obtained by the normalized steepest decent [22]:

$$
\gamma=\frac{\left(\mathbf{Z}^{(q)}\right)^{H} \mathbf{Z}^{(q)}}{\left(\mathbf{Z}^{(q)}\right)^{H} \mathbf{R} \mathbf{Z}^{(q)}},
$$

where $\mathbf{Z}^{(q)}=\mathbf{X}^{H} \mathbf{W}\left(\mathbf{F}-\mathbf{X} \mathbf{a}^{(q)}\right)$ and $\mathbf{R}=\mathbf{X}^{H} \mathbf{W} \mathbf{X}$. $\operatorname{prox}_{\boldsymbol{\ell}}$ is the backward proximity operator which is equivalent to the projection over the excitation constraints $S_{\text {exc }}$.

Here one remark is presented for (11). In the strict sense, the forward and backward splitting iterative process can be applied for convex functions only. When $\ell$ is convex, the forward and backward algorithm converges to the unique solution to (9). When $\ell$ is nonconvex, the convergence of this method is still an open question and the optimum solution cannot be guaranteed [23]. However, despite lack of adequate theoretical justification, the forward and backward 
method is routinely applied to problems in the absence of convexity with good results [24, 25]. Furthermore, when we check (11) in detail, it can be seen that the forward step $\mathbf{P}_{U} \mathbf{a}$ is well-posed because $(1 / 2)\|\mathbf{F}-\mathbf{X a}\|_{\mathbf{W}}^{2}$ is convex. For the backward step $\operatorname{prox}_{\ell}$, it is ill-posed because $\ell$ is nonconvex and the projection over $S_{\text {exc }}$ is not unique. In this case, we should define a fixed rule for the projector over $S_{\text {exc }}$, which is described as follows.

We first define an operator $\mathbf{P}_{\text {exc }}^{\beta}$, which is the projector over $S_{\text {exc }}^{\beta}$ for a fixed scaling factor $\beta$ :

$$
\mathbf{P}_{\mathrm{exc}}^{\beta}=\mathbf{P}_{\text {phase }} \mathbf{P}_{\mathrm{amp}}^{\beta} \text {, }
$$

where $\mathbf{P}_{\text {amp }}^{\beta}$ and $\mathbf{P}_{\text {phase }}$ force the excitation coefficients to satisfy the amplitude constraint $\beta A_{\min } \leq\left|a_{n}\right| \leq \beta A_{\max }$ and phase constraint $\phi_{\min } \leq \arg \left(a_{n}\right) \leq \phi_{\max }$, respectively:

$$
\begin{gathered}
\mathbf{P}_{\mathrm{amp}}^{\beta}: a_{n} \longrightarrow \begin{cases}\beta A_{\max } \frac{a_{n}}{\left|a_{n}\right|}, & \left|a_{n}\right|>\beta A_{\max } \\
a_{n}, & \beta A_{\min } \leq\left|a_{n}\right| \leq \beta A_{\max } \\
\beta A_{\min } \frac{a_{n}}{\left|a_{n}\right|}, & \left|a_{n}\right|<\beta A_{\min },\end{cases} \\
\mathbf{P}_{\text {phase }}: a_{n} \longrightarrow \begin{cases}\left|a_{n}\right| e^{j \phi_{\max },} & \arg \left(a_{n}\right)>\phi_{\max } \\
a_{n}, & \phi_{\min } \leq \arg \left(a_{n}\right) \leq \phi_{\max } \\
\left|a_{n}\right| e^{j \phi_{\min },} & \arg \left(a_{n}\right)<\phi_{\min },\end{cases}
\end{gathered}
$$

Letting $\mathbf{a}^{\beta}=\mathbf{P}_{\operatorname{exc}}^{\beta} \mathbf{a}$, we have the projector of a over $S_{\mathrm{exc}}$ such that

$$
\mathbf{P}_{\mathrm{exc}}: \mathbf{a} \longrightarrow \mathbf{a}^{\widetilde{\beta}}=\mathbf{P}_{\mathrm{exc}}^{\widetilde{\beta}} \mathbf{a},
$$

where $\widetilde{\beta}=\arg \min _{\beta}\left\|\mathbf{a}-\mathbf{a}^{\beta}\right\|_{2}$, which can be calculated using Algorithm 2.

Algorithm 2 (iterative procedure for optimum excitation scaling factor $\widetilde{\beta})$.

Step 1. Initialize the scaling factor $\beta$.

$$
e\left(\theta_{k}, \varphi_{k}\right)= \begin{cases}|| f\left(\theta_{k}, \varphi_{k}\right)\left|-\widetilde{\alpha} M_{L}\left(\theta_{k}, \varphi_{k}\right)\right|, & \left|f\left(\theta_{k}, \varphi_{k}\right)\right|<\widetilde{\alpha} M_{L}\left(\theta_{k}, \varphi_{k}\right) \\ || f\left(\theta_{k}, \varphi_{k}\right)\left|-\widetilde{\alpha} M_{U}\left(\theta_{k}, \varphi_{k}\right)\right|, & \left|f\left(\theta_{k}, \varphi_{k}\right)\right|>\widetilde{\alpha} M_{U}\left(\theta_{k}, \varphi_{k}\right) \\ 0, & \tilde{\alpha} M_{L}\left(\theta_{k}, \varphi_{k}\right) \leq\left|f\left(\theta_{k}, \varphi_{k}\right)\right| \leq \widetilde{\alpha} M_{U}\left(\theta_{k}, \varphi_{k}\right),\end{cases}
$$

where $\tilde{\alpha}$ is the optimum scaling factor determined by Algorithm 1. Then the weights are adjusted according to the differences in each direction, which is expressed by

$$
w^{\text {new }}\left(\theta_{k}, \varphi_{k}\right)=w^{\text {old }}\left(\theta_{k}, \varphi_{k}\right) \frac{2}{1+\exp \left\{-e\left(\theta_{k}, \varphi_{k}\right) / \lambda\right\}},
$$

Step 2. Obtain $\mathbf{a}^{\beta}$ by carrying on the projector $\mathbf{P}_{\mathrm{exc}}^{\beta}$.

Step 3. Calculate the relative error $\delta=\left(\mathbf{a}_{r}^{\beta}-\mathbf{a}_{r}\right)^{T} \mathbf{a}_{r}^{\beta} /\left\|\mathbf{a}_{r}^{\beta}\right\|_{2}^{2}$.

Step 4. Update the scaling factor by $\beta=\beta(1-\delta)$.

Step 5. If convergent or equal to the maximum iteration time, then $\widetilde{\beta}=\beta$ and break; else return to Step 2 .

Here we also use $l_{2}$ norm to compute the optimum scaling factors $\widetilde{\beta}$ and denote $\mathbf{a}_{r}=\left([\operatorname{Re}\{\mathbf{a}\}]^{T},[\operatorname{Im}\{\mathbf{a}\}]^{T}\right)^{T}$ and $\mathbf{a}_{r}^{\beta}=$ $\left(\left[\operatorname{Re}\left\{\mathbf{a}^{\beta}\right\}\right]^{T},\left[\operatorname{Im}\left\{\mathbf{a}^{\beta}\right\}\right]^{T}\right)^{T}$. Thus the iterative process of (11) can be rewritten as

$$
\mathbf{a}^{(q+1)}=\mathbf{P}_{\mathrm{exc}} \mathbf{P}_{U} \mathbf{a}^{(q)}
$$

and the operator $\mathbf{P}_{\text {CWLMS }}$ can be approximated by

$$
\mathbf{P}_{\mathrm{CWLMS}}=\underbrace{\mathbf{P}_{\mathrm{exc}} \mathbf{P}_{U} \cdots \mathbf{P}_{\mathrm{exc}} \mathbf{P}_{U}}_{\mathrm{P}}
$$

where $Q$ is the number of iterations for solving (9). For the initialization of the excitation in (16), we set $\mathbf{a}^{(0)}=$ $\left(\mathbf{X}^{H} \mathbf{W X}\right)^{-1} \mathbf{X}^{H} \mathbf{W F}$. The way of initialization is very efficient and has good convergence.

After the operation of $\mathbf{P}_{\text {CWLMS }}$, a new excitation $\mathbf{a}$ is obtained. Then we can transform a to a new field pattern $\mathbf{F}$ by applying a linear transform $\mathbf{P}_{\mathrm{lt}}: \mathbf{a} \rightarrow \mathbf{F}=\mathbf{X a}$. Therefore, the whole alternating projections can be expressed by

$$
\mathbf{F}_{j+1}=\mathbf{P}_{\mathrm{lt}} \mathbf{P}_{\mathrm{CWLMS}} \mathbf{P}_{\mathrm{patt}} \mathbf{F}_{j}
$$

where $j$ is the iteration time of AP.

3.4. Selection of the Weighted Matrix. In this subsection, the choosing of weighted matrix $\mathbf{W}$ is discussed. Similar to [15, 16], we first calculate the overranging differences for different directions between the obtained pattern after the operation of $\mathbf{P}_{\mathrm{lt}}$ and the upper mask or lower mask at each iteration; that is,

$$
\sum_{k=1}^{K} w^{\text {new }}\left(\theta_{k}, \varphi_{k}\right)=1
$$

where $w^{\text {old }}\left(\theta_{k}, \varphi_{k}\right)$ is the used weight at previous iteration, $w^{\text {new }}\left(\theta_{k}, \varphi_{k}\right)$ represents the adjusted weight, and 


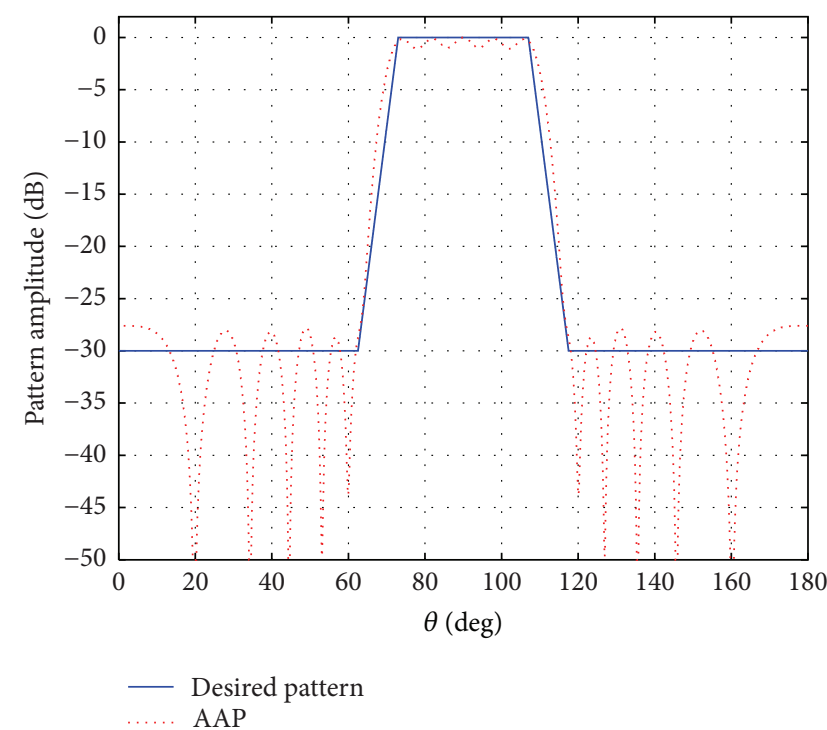

(a)

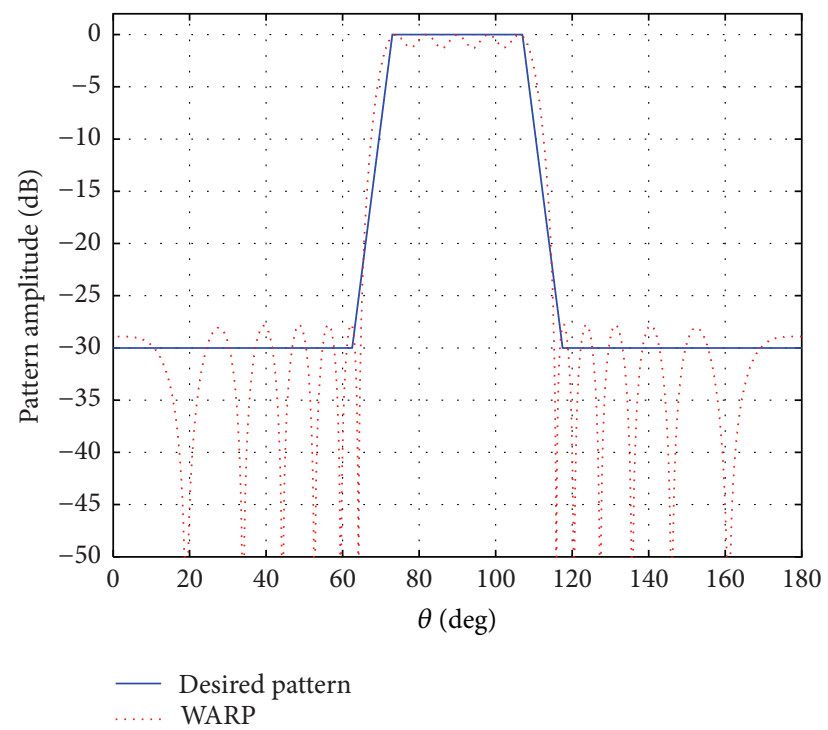

(b)

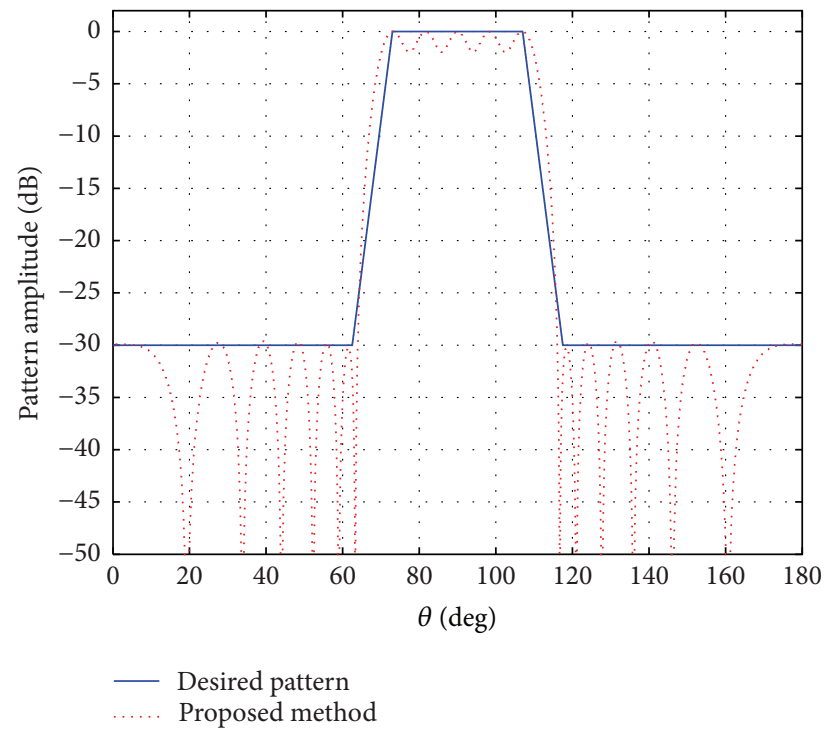

(c)

FIGURe 1: Pattern of FTP synthesis. (a) AAP method, (b) WARP method, and (c) proposed method.

$2 /\left(1+\exp \left\{-e\left(\theta_{k}, \varphi_{k}\right) / \lambda\right\}\right)$ is an S-shape function with the scale parameter $\lambda$. The purpose of $\sum_{k=1}^{K} w^{\text {new }}\left(\theta_{k}, \varphi_{k}\right)=1$ is to regularize the sum of weights in different directions to be 1 , which can avoid the large variation of $\|\mathbf{a}\|_{2}$ and improve the stability of weighted alternating projection.

3.5. Algorithm Summary. The proposed array synthesis method is summarized in Algorithm 3.

Algorithm 3 (scalable array synthesis using weighted alternating projection and proximal splitting).

Step 1. Initialize the field pattern $\mathbf{F}$ and matrix $\mathbf{W}$.
Step 2. Compute the optimum pattern scaling factor $\widetilde{\alpha}$.

Step 3. Impose the scalable pattern constraint using $\mathbf{P}_{\text {patt }}=$ $\mathbf{P}_{\text {patt }}^{\widetilde{\alpha}}$.

Step 4. Calculate the error value and update W.

Step 5. Solve the constrained weighted LMS using $\mathbf{P}_{\text {CWLMS }}$.

Step 5.1. Initialize excitation a.

Step 5.2. Carry on the forward iteration using $\mathbf{P}_{U}$.

Step 5.3. Compute the optimum excitation scaling factor $\widetilde{\beta}$. 


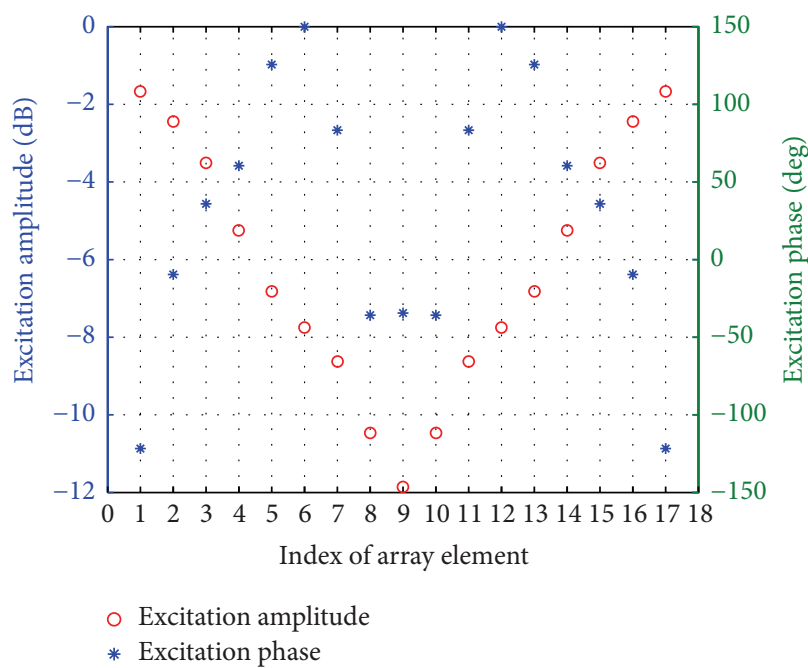

(a)

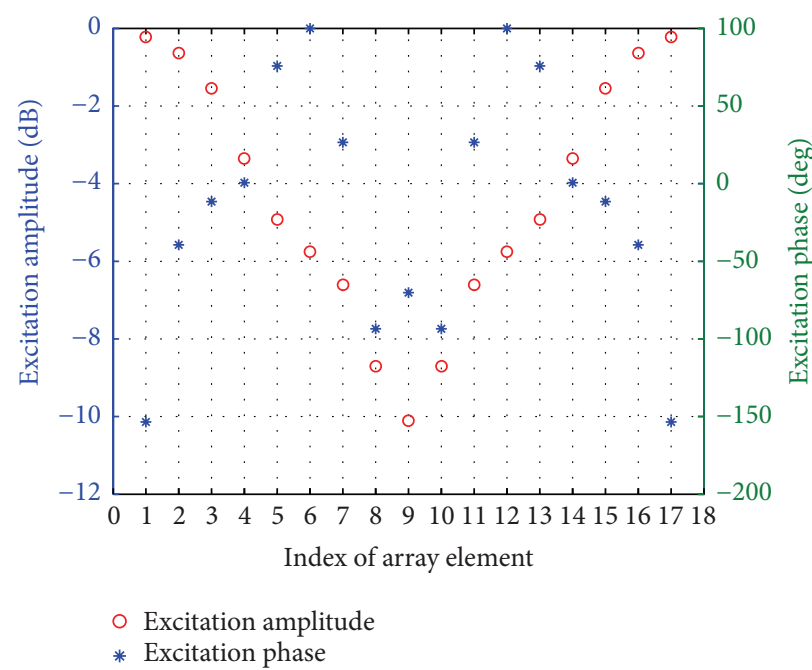

(b)

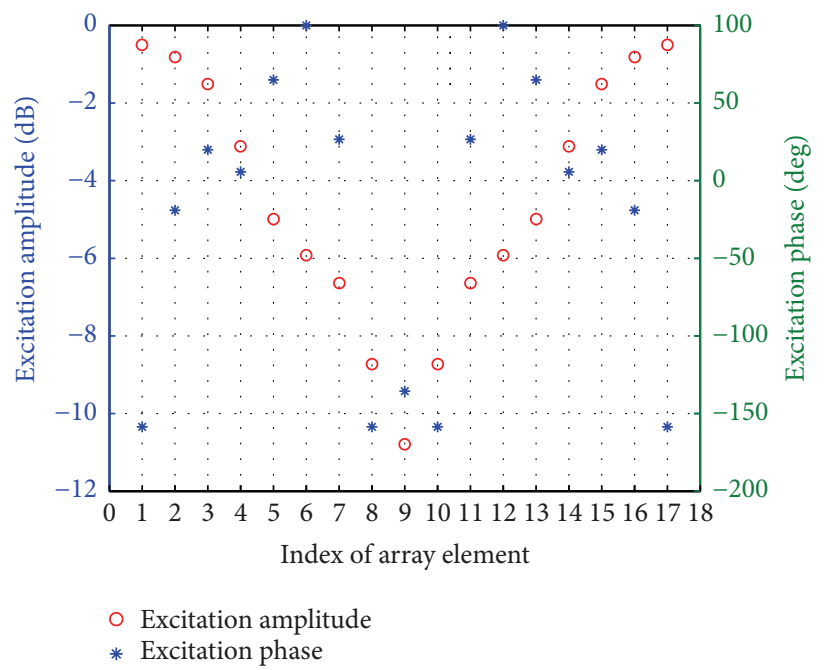

(c)

FIGURE 2: Excitation distributions of FTP synthesis. (a) AAP method, (b) WARP method, and (c) proposed method.

Step 5.4. Impose the scalable excitation constraints using $\mathbf{P}_{\mathrm{exc}}=\mathbf{P}_{\mathrm{exc}}^{\widetilde{\beta}}$.

Step 5.5. If convergent, then break; else return to Step 5.2.

Step 6. Calculate field pattern using $\mathbf{P}_{\mathrm{lt}}$.

Step 7. If $\mathbf{F}$ satisfies the pattern and excitation constraints, then stop; else return to Step 2.

Now the computational complexity of Algorithm 3 is briefly discussed. According to Algorithm 3, Table 1 lists the computational complexity for each step. From Table 1, we can see that the complexity of our algorithm is about

$$
\begin{aligned}
\mathrm{O}\{ & \left\{\left[\max \left(C_{\text {exp }}, N, A\right) K\right.\right. \\
& \left.\left.+\max \left(K N, N^{2}, B C_{\exp }\right) N Q\right] Z\right\},
\end{aligned}
$$

TABLE 1: The computational complexity of proposed array synthesis algorithm.

\begin{tabular}{lc}
\hline Terms to compute & Computational complexity \\
\hline Compute $\tilde{\alpha}$ and $\mathbf{P}_{\text {patt }}^{\tilde{\alpha}}$ & $\mathrm{O}(K A)$ \\
Update $\mathbf{W}$ & $\mathrm{O}\left(K C_{\text {exp }}\right)$ \\
$\mathbf{P}_{\text {CWLMS }}$ initialize a & $\mathrm{O}\left[N^{2} \max (K, N)\right]$ \\
$\mathbf{P}_{\text {CWLMS }}:$ compute $\mathbf{P}_{U}$ & $\mathrm{O}(K N)$ \\
$\mathbf{P}_{\text {CWLMS }}:$ compute $\tilde{\beta}$ and $\mathbf{P}_{\text {exc }}^{\widetilde{\beta}}$ & $\mathrm{O}\left(N B C_{\text {exp }}\right)$ \\
Compute $\mathbf{P}_{\text {lt }}$ & $\mathrm{O}(K N)$ \\
\hline
\end{tabular}

where $Z$ and $Q$ are the numbers of outer and inner iterations of AP. $A$ and $B$ are the iteration times of computing the 


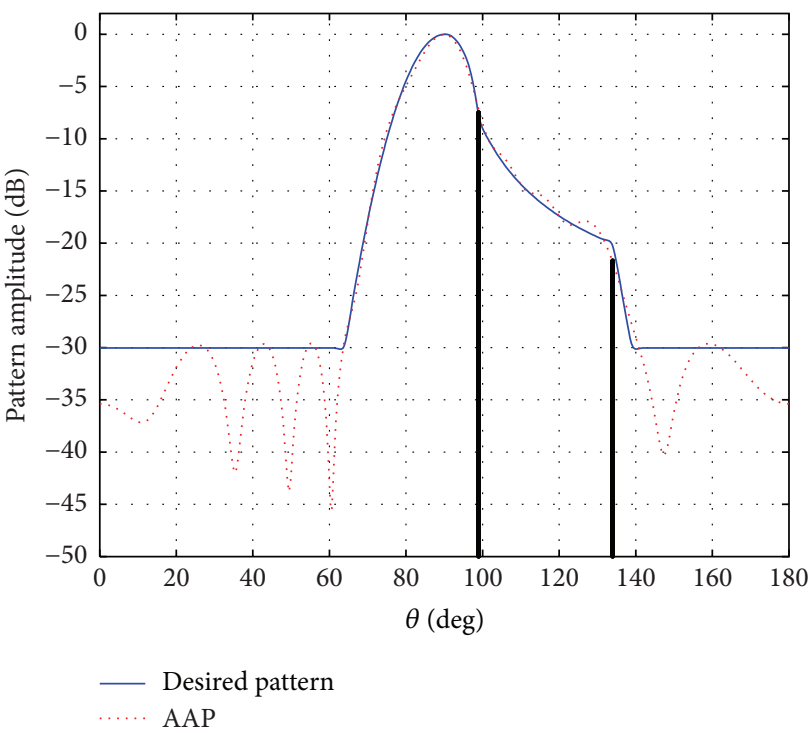

(a)

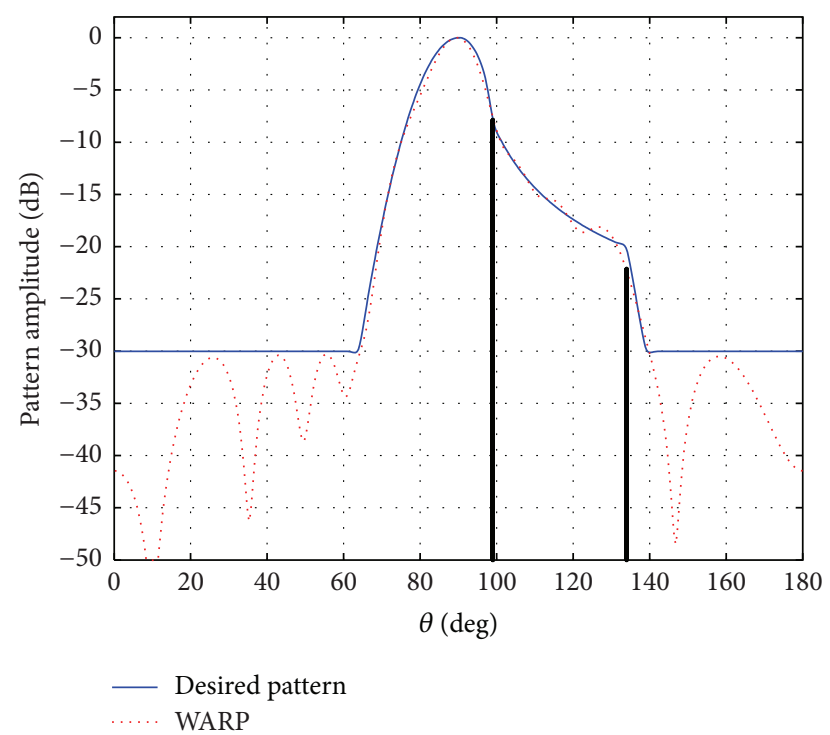

(b)

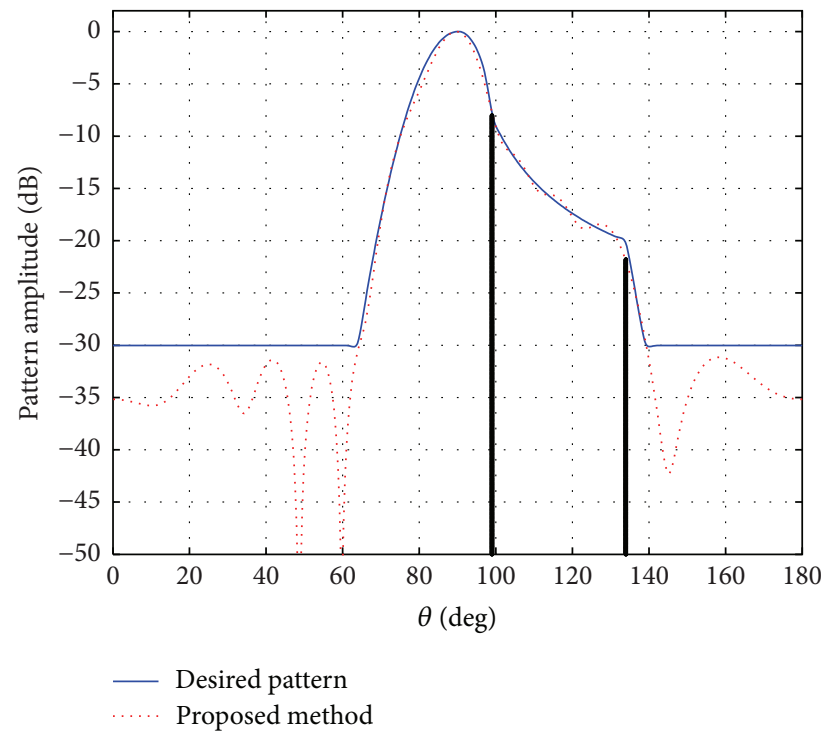

(c)

FIgURE 3: Pattern of CSP synthesis. (a) AAP method, (b) WARP method, and (c) proposed method.

optimum pattern and excitation scaling factors. $N$ and $K$ are the numbers of array elements and pattern sampling directions. $C_{\exp }$ is the exponential complexity which arises from the calculations of weighted matrix $\mathbf{W}$ and operator $\mathbf{P}_{\text {phase }}$. It is worth mentioning that the number of inner iterations $Q$ does not need to be very large to ensure the convergence of inner iteration. A small value can be set to reduce the oscillations in iterative process and accelerate the convergence of AP method [26]. For the iteration times of $A$ and $B$, from the statement in [13], we can know that few iterations should suffice in approaching the $l_{2}$ norm exact projection to find the optimum scaling factors. So they can be set to be small values which leads to negligible increase in the computational cost compared to the fixed scaling factors.

\section{Synthesis Examples}

To demonstrate the performance of proposed method, some synthesis examples are provided. In Section 4.1, we consider two examples for the linear array synthesis using a flat-topped pattern (FTP) and a cosecant-squared pattern (CSP). One is with excitation amplitude constraint, and the other is with excitation amplitude and phase constraints. For comparison, we also provided the synthesis results of AAP [13] and WARP [16]. In Section 4.2, three pattern synthesis examples are provided for a planar array with excitation amplitude constraint, whose fluctuation is less than a prescribed value.

4.1. Synthesis with Linear Array. As illustrated in Figure 1, we first synthesize an FTP whose shaped region is from 




(a)

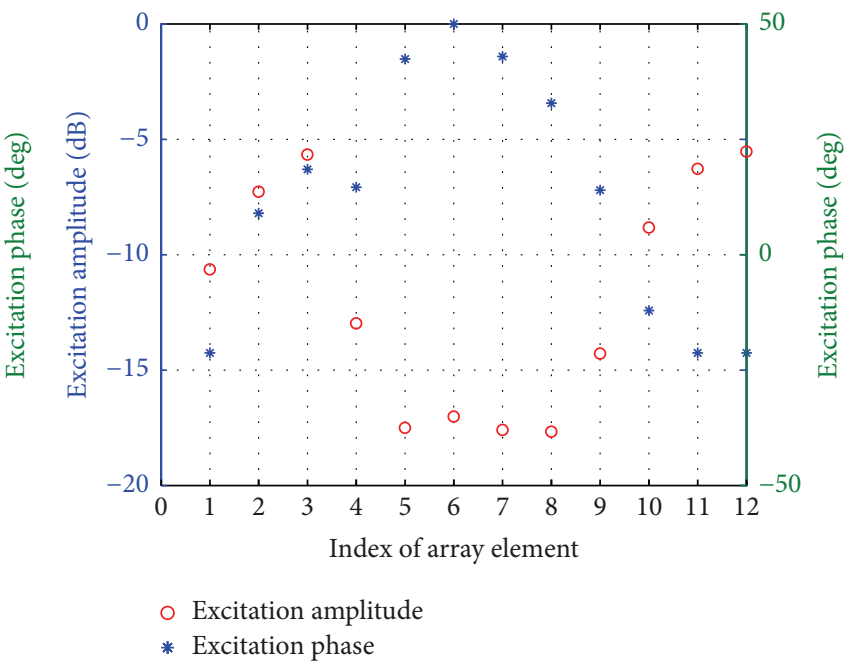

(b)

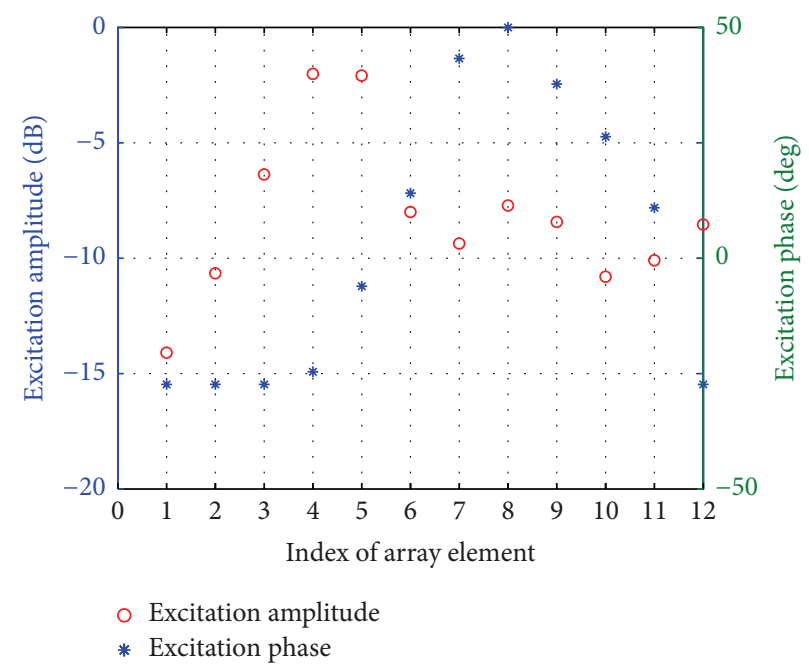

(c)

FIGURE 4: Excitation distributions of CSP synthesis. (a) AAP method, (b) WARP method, and (c) proposed method.

$73^{\circ}$ to $107^{\circ}$. A uniform linear array with 17 omnidirectional elements spaced half a wavelength apart is considered. The ripples are required to be less than $2 \mathrm{~dB}$ in the shaped regions and the SLL is lower than $-30 \mathrm{~dB}$. The excitation amplitude constraints are $A_{\max } / A_{\min }=3.5(10.8814 \mathrm{~dB})$ and there is no phase constraint. The synthesis results of AAP, WARP, and our proposed method are given in Figures 1(a), 1(b), and 1(c), respectively. Figures 2(a), 2(b), and 2(c) are the excitation amplitude and phase distributions for different methods. The excitations for our synthesised FTP are especially provided in Table 2. The comparisons of maximum SLL and mainlobe ripple have been listed in Table 3. From Figures 1 and 2 and Table 3 , we can see that the excitation amplitude dynamics are all less than 3.5. Our synthesis result shows a very good fit to destination pattern. The ripple level in the shaped region is about $1.97 \mathrm{~dB}$ and the maximum SLL is $-29.6 \mathrm{~dB}$, which is slightly higher than $-30 \mathrm{~dB}$, but much lower than AAP and WARP methods.
Next we consider the CSP synthesis with excitation amplitude and phase constraints. As shown in Figure 3, the shaped region is from $99^{\circ}$ to $134^{\circ}$ and the SLL is also required to be lower than $-30 \mathrm{~dB}$. The proposed method is examined with a 12 -element linear array spaced half a wavelength apart. The excitation amplitude and phase constraints are $A_{\max } / A_{\min }=$ $6.5(16.2583 \mathrm{~dB})$ and $\phi_{\max }=-\phi_{\min }=50^{\circ}$. Figures 3(a), 3(b), and $3(\mathrm{c})$ are the synthesized patterns of AAP, WARP, and our proposed method, respectively. Figures 4(a), 4(b), and $4(c)$ are the excitation amplitude and phase distributions for different methods. The excitations of our synthesised CSP pattern are shown in Table 2. The values of maximum SLL and mainlobe ripples are also compared in Table 3. From Figures 3 and 4 and Table 3 , it can be observed that the obtained excitations of three methods are all satisfied to the excitation amplitude and phase constraints. The proposed method provides better performance compared to WARP and 




(a)

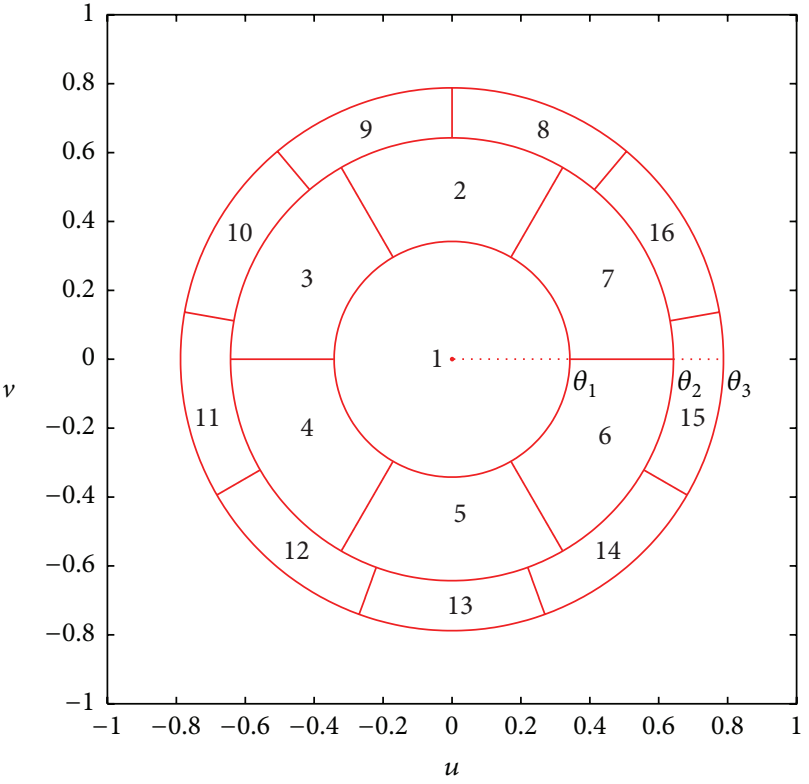

(b)

FiguRE 5: Array arrangement and pattern coverage regions. (a) The arrangement of the 91-element array and (b) the coverage regions of 16 different patterns.

TABLE 2: Excitations for our synthesised FTP and CSP patterns.

\begin{tabular}{|c|c|c|c|c|c|c|c|c|c|}
\hline $\operatorname{FTP}(n)$ & 1 & 2 & 3 & 4 & 5 & 6 & 7 & 8 & 9 \\
\hline$\left|a_{n}\right|$ & 0.3040 & 0.5779 & 0.6919 & 0.6476 & 0.8505 & 1.0000 & 0.7131 & 0.3040 & 0.3379 \\
\hline$\phi_{n}\left({ }^{\circ}\right)$ & 87.4214 & 79.4566 & 62.2412 & 22.0796 & -24.7133 & -48.0413 & -65.9425 & -118.1336 & -169.8769 \\
\hline $\operatorname{FTP}(n)$ & 10 & 11 & 12 & 13 & 14 & 15 & 16 & 17 & \\
\hline$\left|a_{n}\right|$ & 0.3040 & 0.7131 & 1.0000 & 0.8505 & 0.6476 & 0.6919 & 0.5779 & 0.3040 & \\
\hline$\phi_{n}\left({ }^{\circ}\right)$ & -118.1336 & -65.9425 & -48.0413 & -24.7133 & 22.0796 & 62.2412 & 79.4566 & 87.4214 & \\
\hline $\operatorname{CSP}(n)$ & 1 & 2 & 3 & 4 & 5 & 6 & & & \\
\hline$\left|a_{n}\right|$ & 0.1684 & 0.1684 & 0.1684 & 0.1794 & 0.2748 & 0.4371 & & & \\
\hline$\phi_{n}\left({ }^{\circ}\right)$ & -20.4395 & -3.2818 & 18.1418 & 39.8858 & 39.5349 & 10.0440 & & & \\
\hline $\operatorname{CSP}(n)$ & 7 & 8 & 9 & 10 & 11 & 12 & & & \\
\hline$\left|a_{n}\right|$ & 0.8563 & 1.0000 & 0.7537 & 0.5797 & 0.4067 & 0.1684 & & & \\
\hline$\phi_{n}\left({ }^{\circ}\right)$ & 3.2090 & 11.3957 & 7.8043 & -4.0307 & -0.4343 & 7.2796 & & & \\
\hline
\end{tabular}

TABLE 3: Comparison of different methods for FTP and CSP syntheses.

\begin{tabular}{|c|c|c|c|c|c|}
\hline Patterns & Excitation constraints & Characteristics of patterns $(\mathrm{dB})$ & AAP & WARP & Proposed \\
\hline \multirow{2}{*}{$\operatorname{FTP}(N=17)$} & \multirow{2}{*}{$\frac{A_{\max }}{A_{\min }}=3.5$} & SLL & -27.6 & -27.7 & -29.6 \\
\hline & & Ripple & 1.07 & 1.31 & 1.97 \\
\hline \multirow{2}{*}{$\operatorname{CsP}(N=12)$} & \multirow{2}{*}{$\frac{A_{\max }}{A_{\min }}=6.5, \quad \phi_{\max }=50^{\circ}$} & SLL & -28.9 & -30.3 & -31.2 \\
\hline & & Ripple & 0.83 & 0.81 & 0.75 \\
\hline
\end{tabular}

AAP methods. The ripple level in the shaped region is about $0.75 \mathrm{~dB}$ and the SLLs are lower than $-30 \mathrm{~dB}$.

4.2. Synthesis with Planar Array. In this subsection, an $\mathrm{S}$ band planar array of 91 elements for low earth orbit satellite communication is considered. As illustrated in Figure 5(a), the elements of array form an equilateral triangle arrangement with the interelement spacing of half wavelength. There are 16 different patterns shown in Figure 5(b) to be synthesized in UV plane, where $u=\sin (\theta) \cos (\varphi), v=\sin (\theta) \sin (\varphi)$. The goal is to synthesize these patterns with consideration of 91 embedded element patterns, which are evaluated by 


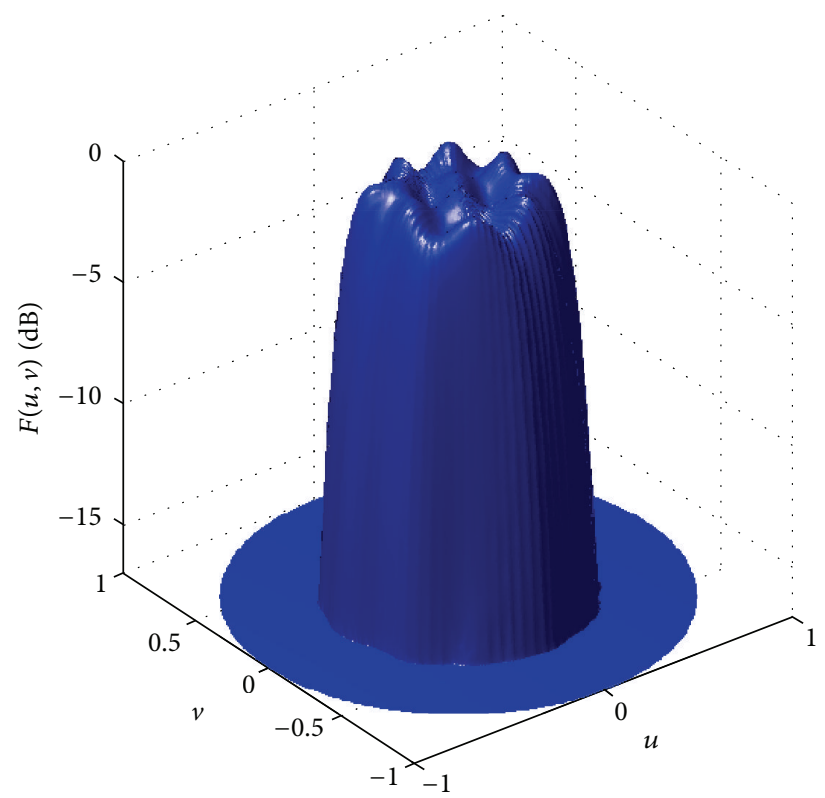

(a)

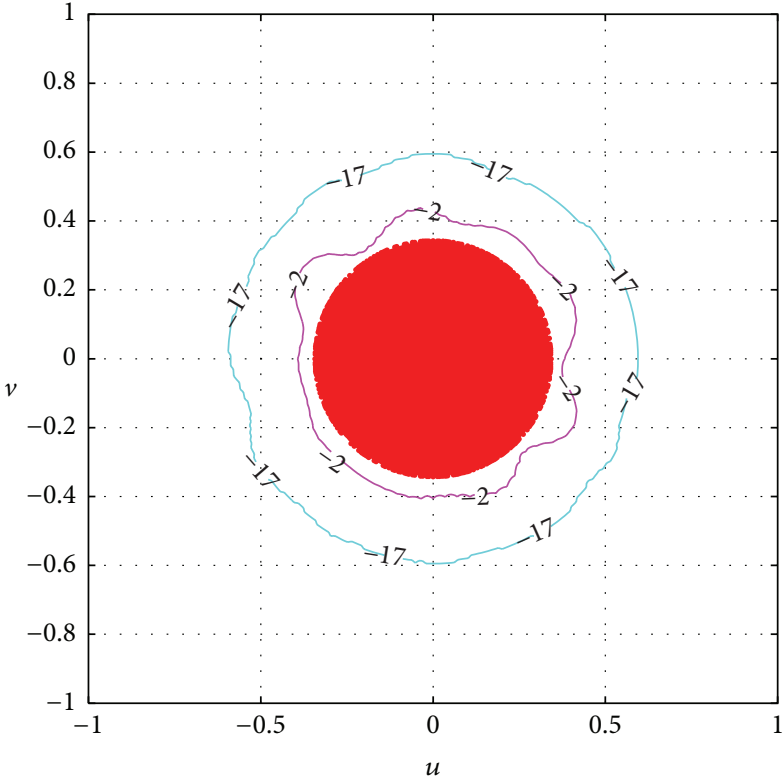

(b)

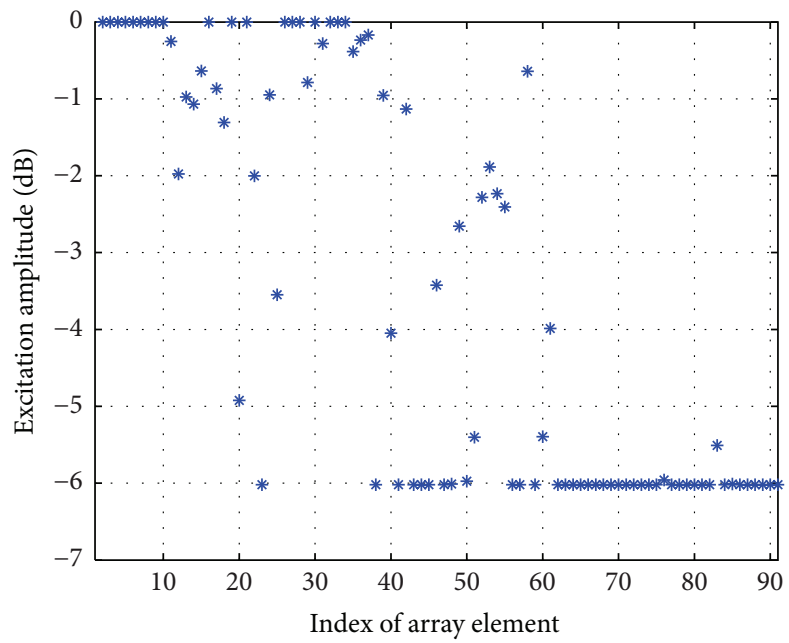

(c)

FIGURE 6: Synthesis result of pattern 1 for planar array. (a) The synthesized pattern, (b) the contour plot, and (c) the normalized excitation amplitude.

using HFSS software. The maximum SLL is required to be lower than $-17 \mathrm{~dB}$ and the ripples in shaped regions must be less than $2 \mathrm{~dB}$. The excitation amplitude dynamics are $A_{\text {max }} / A_{\text {min }}=2(6.0206 \mathrm{~dB})$. For convenience, we select pattern 1 , pattern 2 , and pattern 8 as examples. The shaped region of pattern 1 is a circular footprint located on the center of UV plane with $\theta \in\left[0^{\circ}, \theta_{1}=20^{\circ}\right]$ and $\varphi \in\left[0^{\circ}, 360^{\circ}\right]$, pattern 2 is located in the region of $\theta \in\left[\theta_{1}, \theta_{2}=40^{\circ}\right]$ and $\varphi \in\left[60^{\circ}, 120^{\circ}\right]$, and pattern 8 has an arc-shaped footprint located in $\theta \in\left[\theta_{2}, \theta_{3}=52^{\circ}\right]$ and $\varphi \in\left[50^{\circ}, 90^{\circ}\right]$.

Figures 6, 7, and 8 depict the synthesized results for patterns 1,2, and 8. Figures 6(a), 7(a), and 8(a) are the synthesized patterns, where only the patterns above $-17 \mathrm{~dB}$ are shown for clarity. The contour plots of three patterns with levels of $-2 \mathrm{~dB}$ and $-17 \mathrm{~dB}$ are presented in Figures 6(b), 7(b), and 8(b). The normalized excitation amplitudes of patterns 1, 2, and 8 are plotted in Figures 6(c), 7(c), and 8(c). From Figures 6, 7, and 8, it can be observed that the $-2 \mathrm{~dB}$ contours all fully cover the shaped regions and the ripples in these regions are less than $2 \mathrm{~dB}$. The maximum SLLs are all lower than $-17 \mathrm{~dB}$. The excitation amplitude dynamics for patterns 1,2 , and 8 have been successfully controlled to satisfy $A_{\text {max }} / A_{\text {min }}=2$.

\section{Conclusions}

In this paper, an array pattern synthesis method combining scalable alternating projection and proximal splitting is developed. In this method, the embedded element patterns and 


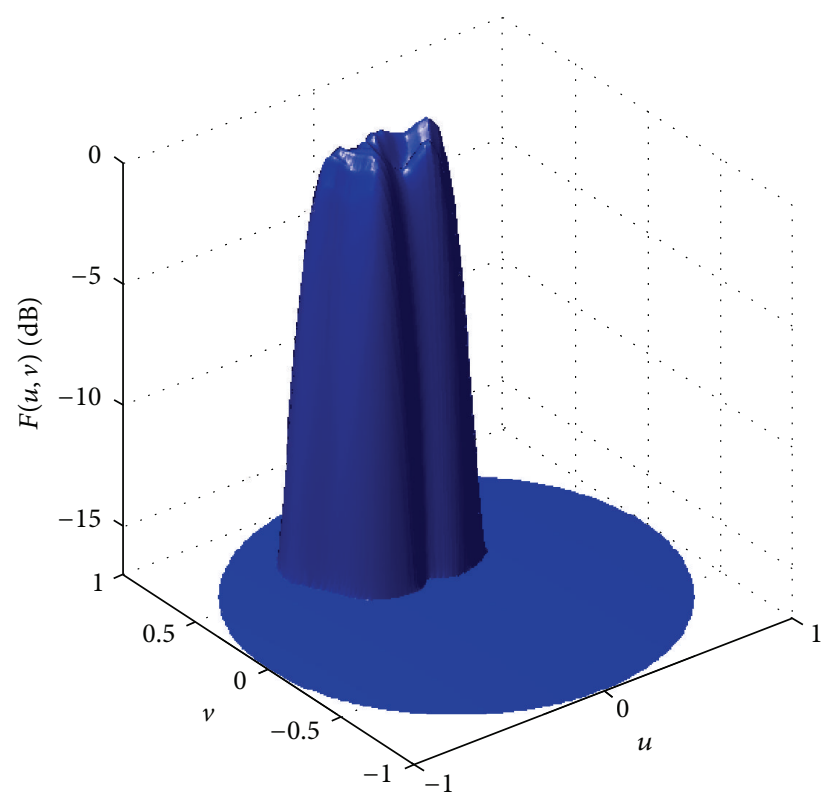

(a)

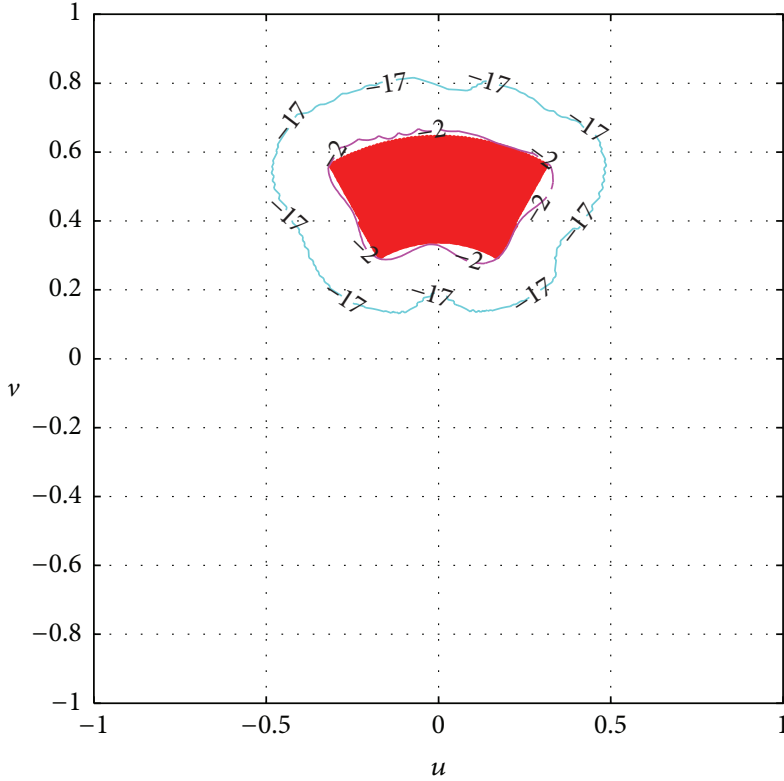

(b)

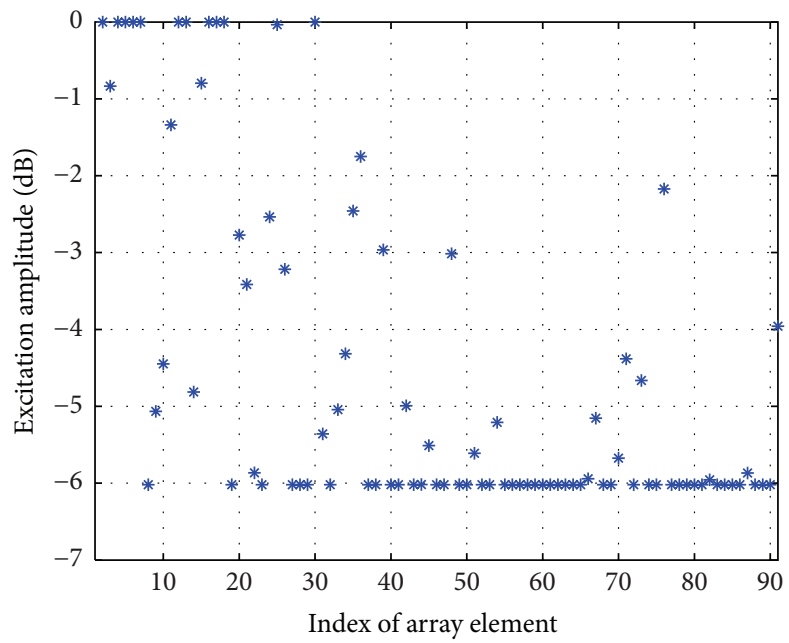

(c)

FIGURE 7: Synthesis result of pattern 2 for planar array. (a) The synthesized pattern, (b) the contour plot, and (c) the normalized excitation amplitude.

scaling invariance property of design specifications are considered. Compared to other alternating projection methods, the proposed array pattern synthesis designs the projector over scalable excitation constraint set as the solution to a CWLMS problem which is solved using forward-backward proximal splitting. Several linear and planar synthesis examples demonstrate the better performance of the proposed array pattern synthesis.

\section{Abbreviations}

AP: Alternating projection

LMS: Least mean squares
WLMS: Weighted least mean squares

AAP: $\quad$ Alternating adaptive projections

WARP: Weighted alternating reverse projection

CWLMS: Constrained weighted least mean squares

FTP: $\quad$ Flat-topped pattern

CSP: Cosecant-squared pattern

SLL: $\quad$ Sidelobe level.

\section{Conflict of Interests}

The authors declare that there is no conflict of interests regarding the publication of this paper. 


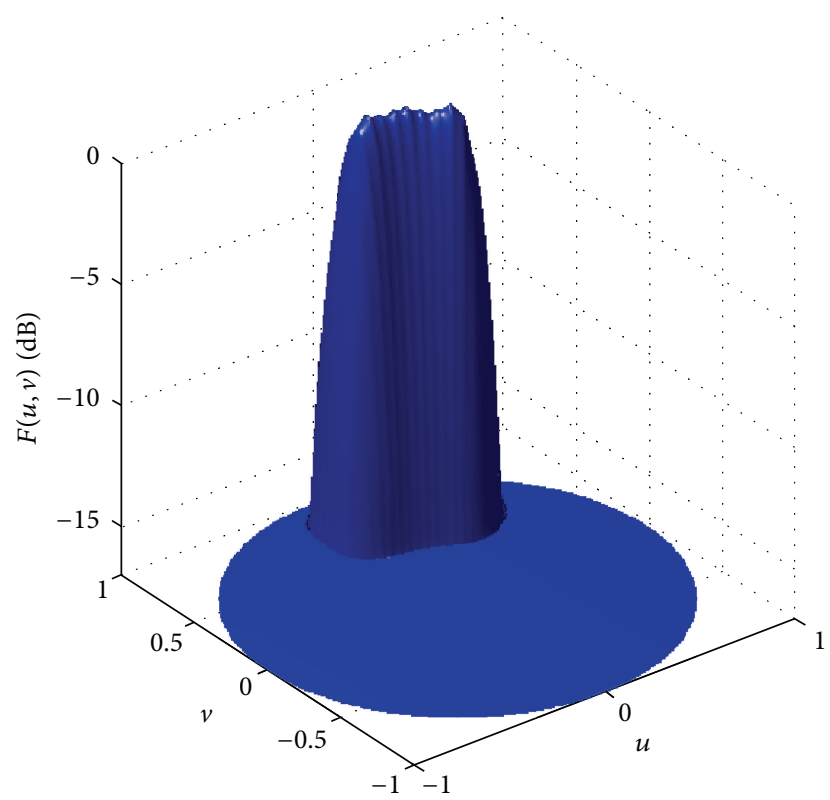

(a)

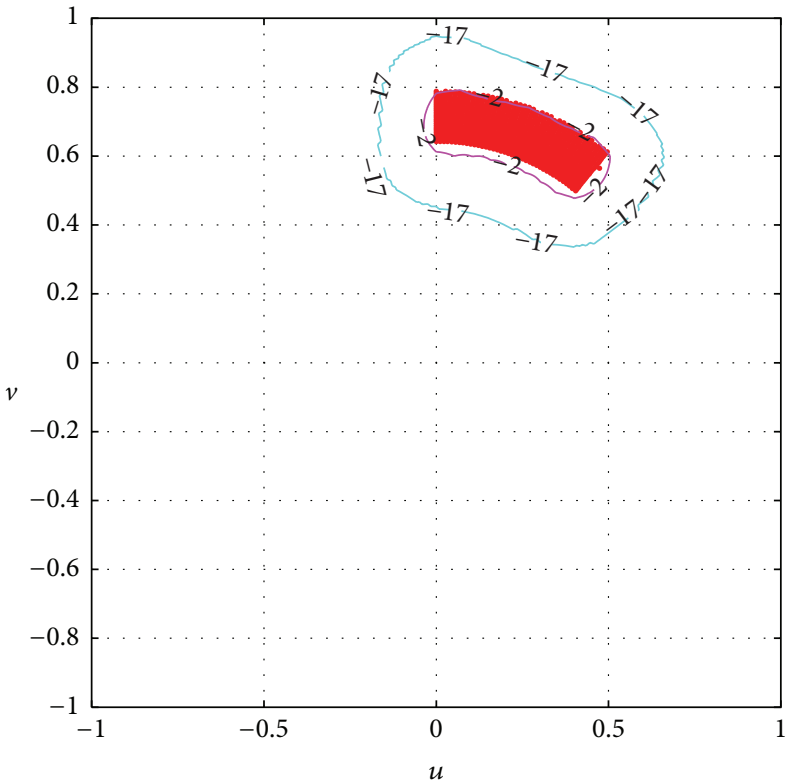

(b)

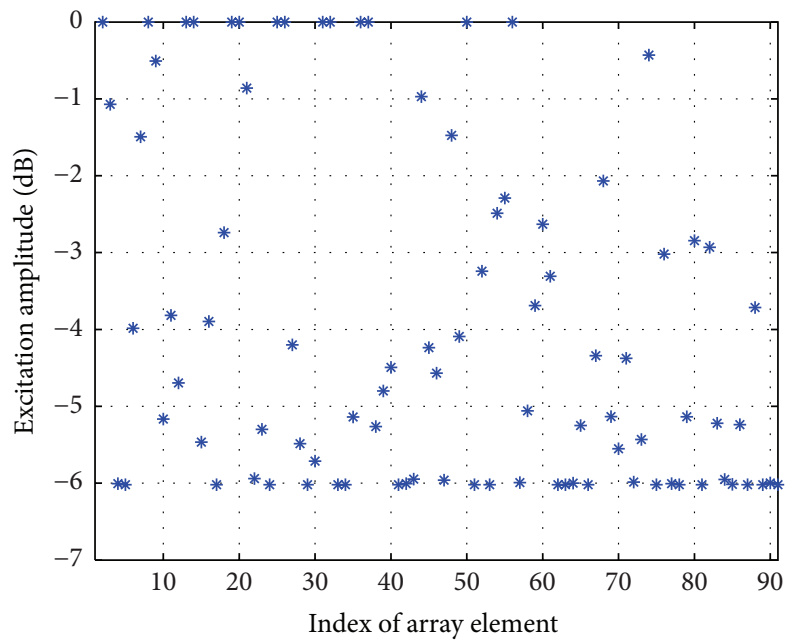

(c)

FIGURE 8: Synthesis result of pattern 8 for planar array. (a) The synthesized pattern, (b) the contour plot, and (c) the normalized excitation amplitude.

\section{Acknowledgment}

This study is supported in part by the National Natural Science Foundations of China (nos. 11273017 and 61471196).

\section{References}

[1] C. A. Balanis, Antenna Theory: Analysis and Design, Wiley, New York, NY, USA, 3rd edition, 2005.

[2] G. A. Somers, "Proof of the Woodward-Lawson sampling method for a finite linear array," Radio Science, vol. 28, no. 4, pp. 481-485, 1993.

[3] A. T. Villeneuve, "Taylor patterns for discrete arrays," IEEE Transactions on Antennas and Propagation, vol. 32, no. 10, pp. 1089-1093, 1984.
[4] C. L. Dolph, "A current distribution for broadside arrays which optimizes the relationship between beam width and side-lobe level," Proceedings of the IRE, vol. 34, no. 6, pp. 335-348, 1946.

[5] H. M. Elkamchouchi and M. M. Hassan, "Array pattern synthesis approach using a genetic algorithm," IET Microwaves, Antennas and Propagation, vol. 8, no. 14, pp. 1236-1240, 2014.

[6] F. Zhang, W. Jia, and M. Yao, "Linear aperiodic array synthesis using differential evolution algorithm," IEEE Antennas and Wireless Propagation Letters, vol. 12, pp. 797-800, 2013.

[7] J. A. Ferreira and F. Ares, "Pattern synthesis of conformal arrays by the simulated annealing technique," Electronics Letters, vol. 33, no. 14, pp. 1187-1189, 1997.

[8] R. K. Baee, K. Forooraghi, and S. Chamaani, "Conformal array pattern synthesis using a hybrid WARP/2LB-MOPSO 
algorithm," International Journal of Antennas and Propagation, vol. 2012, Article ID 202906, 7 pages, 2012.

[9] O. M. Bucci, G. D. Elia, and G. Romito, "Intersection approach to array pattern synthesis," IEE Proceedings-Microwaves, Antennas and Propagation, vol. 137, no. 6, pp. 349-357, 1990.

[10] O. M. Bucci, G. D’Elia, and G. Romito, "Power synthesis of conformal arrays by a generalised projection method," IEE Proceedings: Microwaves, Antennas and Propagation, vol. 142, no. 6, pp. 467-471, 1995.

[11] B. D. Carlson and D. Willner, "Antenna pattern synthesis using weighted least squares," IEE Proceedings $H$ : Microwaves, Antennas and Propagation, vol. 139, no. 1, pp. 11-16, 1992.

[12] L. I. Vaskelainen, "Iterative least-squares synthesis methods for conformai array antennas with optimized polarization and frequency properties," IEEE Transactions on Antennas and Propagation, vol. 45, no. 7, pp. 1179-1185, 1997.

[13] J. L. Araque Quijano and G. Vecchi, "Alternating adaptive projections in antenna synthesis," IEEE Transactions on Antennas and Propagation, vol. 58, no. 3, pp. 727-737, 2010.

[14] E. Ercil, E. Yildirim, and M. E. Inal, "Array Antenna pattern synthesis using measured active element patterns and GramSchmidt Orthogonalization," in Proceedings of the 4th IEEE International Symposium on Phased Array Systems and Technology, pp. 357-360, Waltham, Mass, USA, October 2010.

[15] A. Haddadi, A. Ghorbani, and J. Rashed-Mohassel, "Cosecantsquared pattern synthesis using a weighted alternating reverse projection method," IET Microwaves, Antennas \& Propagation, vol. 5, no. 15, pp. 1789-1795, 2011.

[16] R. Karimzadeh, M. Hakkak, A. Haddadi, and K. Forooraghi, "Conformal array pattern synthesis using the weighted alternating reverse projection method considering mutual coupling and embedded-element pattern effects," IET Microwaves, Antennas and Propagation, vol. 6, no. 6, pp. 621-626, 2012.

[17] Y. Han, C. Wan, W. Sheng, B. Tian, and H. Yang, "Array synthesis using weighted alternating projection and proximal splitting," IEEE Antennas and Wireless Propagation Letters, vol. 14, pp. 1006-1009, 2015.

[18] R. Escalante and M. Raydan, Alternating Projection MethodsFundamentals of Algorithms, U.S.: Society for Industrial and Applied Mathematics, 2011.

[19] M. K. Tam, The Method of Alternating Projections, University of Newcastle, Callaghan, Australia, 2012.

[20] P. L. Combettes and V. R. Wajs, "Signal recovery by proximal forward-backward splitting," Multiscale Modeling \& Simulation, vol. 4, no. 4, pp. 1168-1200, 2005.

[21] P. L. Combettes and J.-C. Pesquet, "Proximal splitting methods in signal processing," in Fixed-Point Algorithms for Inverse Problems in Science and Engineering, pp. 185-212, Springer, New York, NY, USA, 2011.

[22] L. A. Hageman and D. M. Young, Applied Iterative Methods, Academic Press, New York, NY, USA, 1981.

[23] J. Bolte, S. Sabach, and M. Teboulle, "Proximal alternating linearized minimization for nonconvex and nonsmooth problems," Mathematical Programming, vol. 146, no. 1-2, pp. 459494, 2014.

[24] H. Attouch, J. Bolte, P. Redont, and A. Soubeyran, "Proximal alternating minimization and projection methods for nonconvex problems: an approach based on the Kurdyka-Łojasiewicz inequality," Mathematics of Operations Research, vol. 35, no. 2, pp. $438-457,2010$.
[25] H. H. Bauschke, H. M. Phan, and X. Wang, "The method of alternating relaxed projections for two nonconvex sets," Vietnam Journal of Mathematics, vol. 42, no. 4, pp. 421-450, 2014.

[26] H. H. Bauschke, D. R. Luke, H. M. Phan, and X. Wang, "Restricted normal cones and the method of alternating projections: theory," Set-Valued and Variational Analysis, vol. 21, no. 3, pp. 431-473, 2013. 





The Scientific World Journal
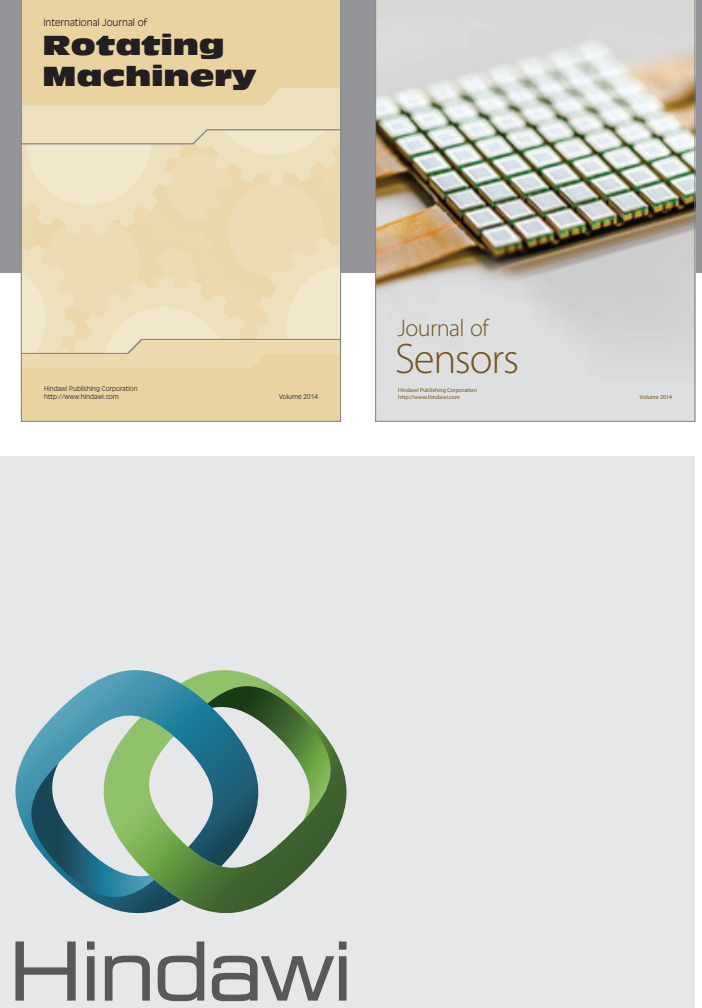

Submit your manuscripts at http://www.hindawi.com
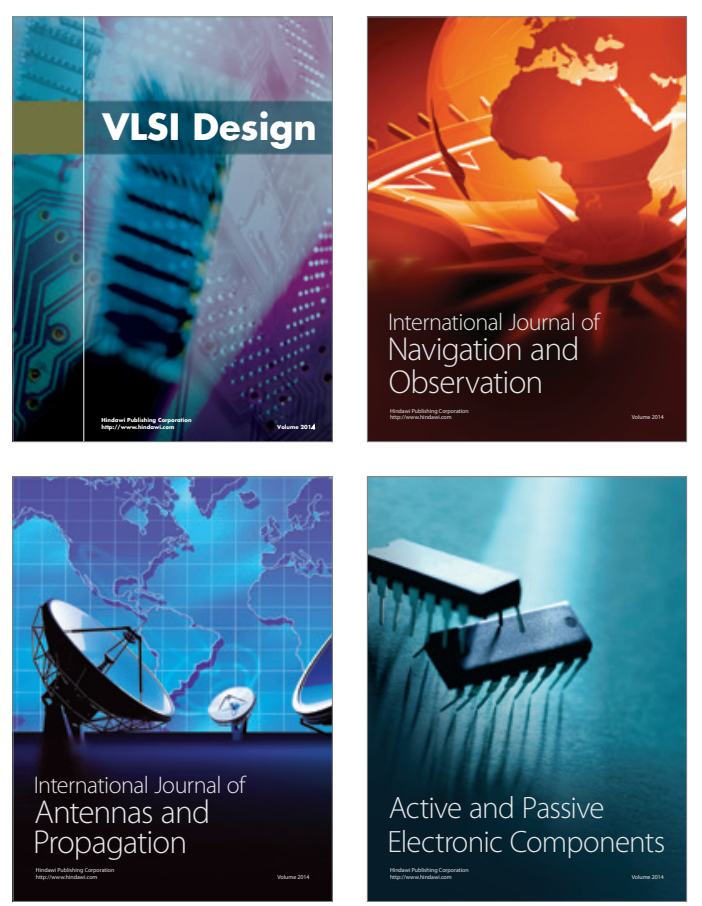
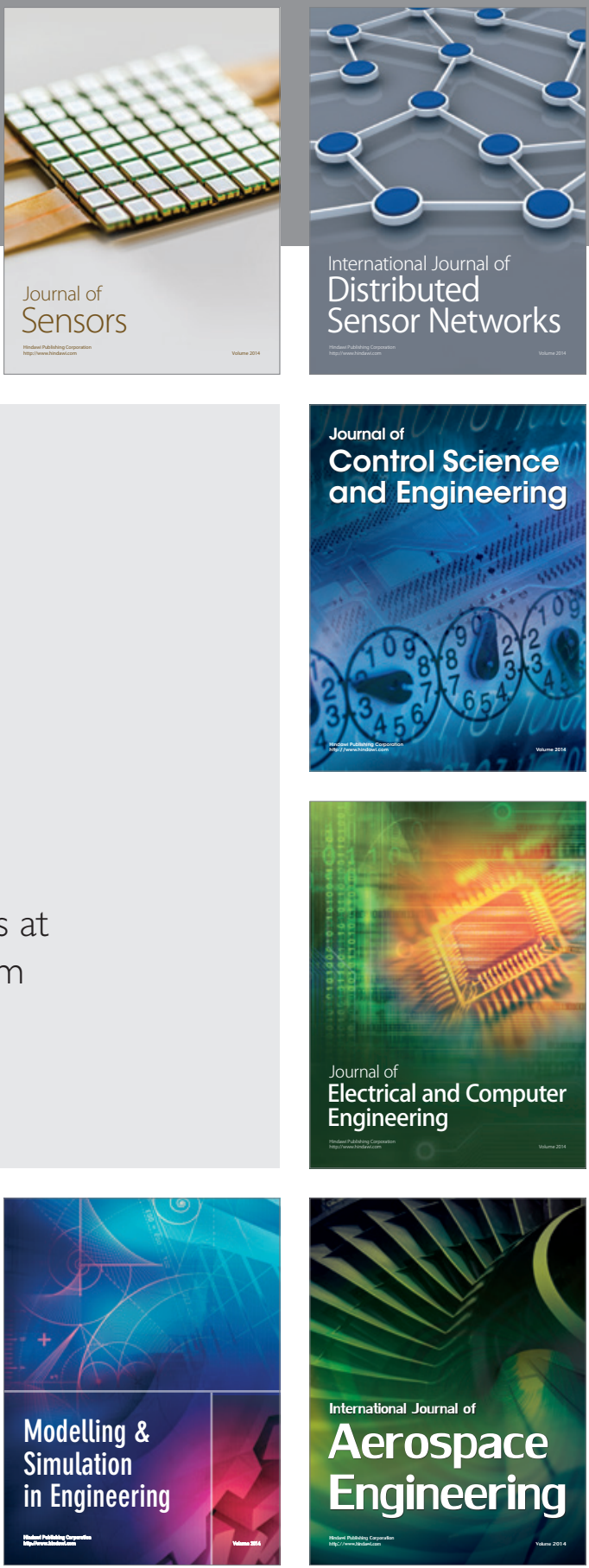

Journal of

Control Science

and Engineering
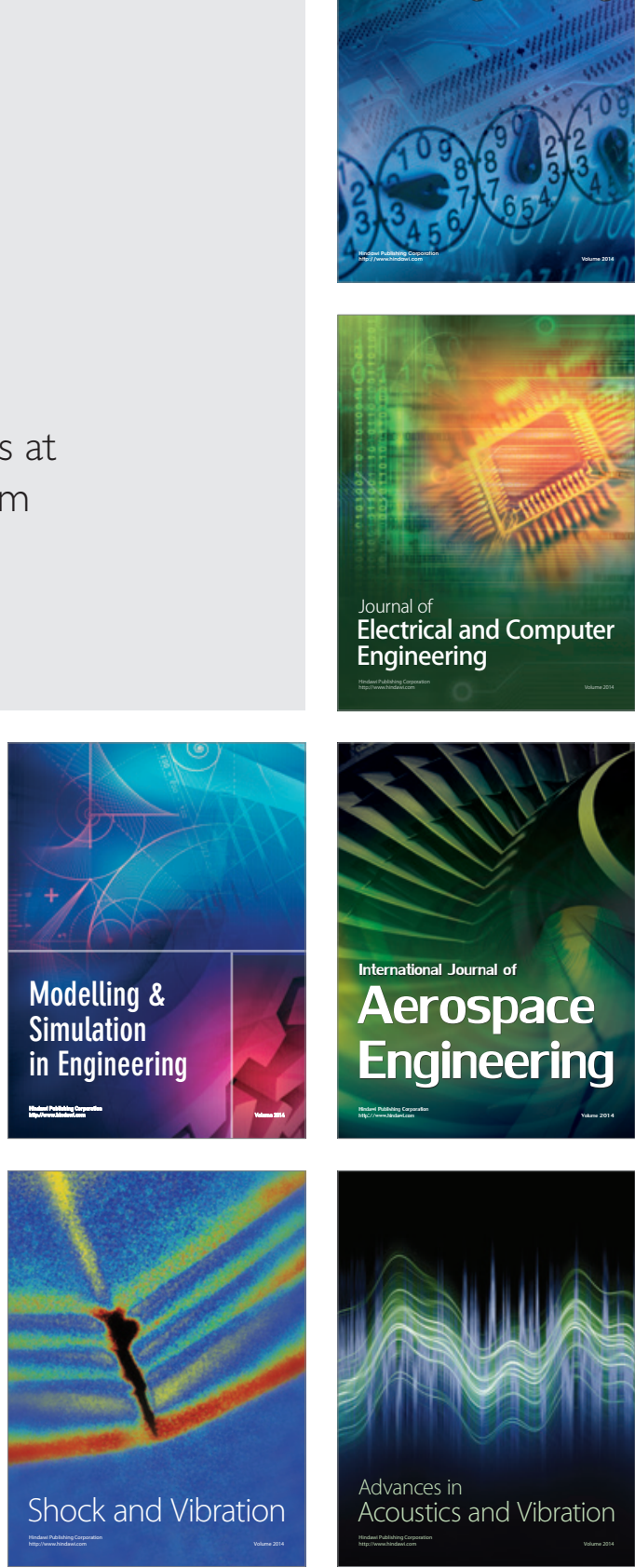